\title{
¿CÓMO HAN AFRONTADO LAS FAMILIAS DE ESTUDIANTES DE EDUCACIÓN PRIMARIA LAS TAREAS ESCOLARS EN EL CONFINAMIENTO DE COVID-19?
}

\author{
Lucía Pérez Vera \\ Universidad de Extremadura \\ luçiapv@unex.es \\ Miguel Ángel Durán Vinagre \\ Universidad de Extremadura \\ mduranv@unex.es \\ Ana Paredes Espinosa \\ Universidad de Extremadura \\ aparedesz@alumnos.unex.es \\ Susana Sánchez Herrera \\ Universidad de Extremadura \\ ssanchez@unex.es
}

Recepción Artículo: 16 mayo 2021 Admisión Evaluación: 16 mayo 2021 Informe Evaluador 1: 27 mayo 2021 Informe Evaluador 2: 02 junio 2021 Aprobación Publicación: 03 junio 2021

\section{Proyecto: Ayuda a grupos de la Junta de Extremadura (GR18132) y FEDER una manera de hacer Europa}

\section{RESUMEN}

Durante el periodo comprendido entre los meses de marzo, abril y mayo de 2020, muchos sectores laborales y formativos se vieron paralizados a consecuencia de la pandemia ocasionada por el COVID-19, viéndose obligados a realizar sus actividades de forma online y por ello, el sistema educativo español tuvo que ejercer el proceso de enseñanza-aprendizaje en un nuevo escenario. Con la finalidad de conocer las percepciones de los familiares de estudiantes de Educación Primaria, en centros privados-concertados, el presente estudio difundió un cuestionario durante el mes de marzo de 2020. Los resultados del análisis de los datos recogidos presentan que la mayoría de las familias de la muestra analizada consideran que la carga de tareas debería variar según las asignaturas, mostrando en su mayoría paciencia en la resolución de dudas y desarrollando una buena planificación para continuar con el desarrollo educativo del curso desde el hogar, manifestando que el tiempo empleado en la realización de las tareas era extenso.

Palabras clave: educación primaria; tareas escolares; familia; Covid-19; percepciones 


\section{ABSTRACT}

Family perception of primary chool students in private centers during Covid-19 confinement. During the period from March, April and May 2020, many labour and training sectors were paralyzed as a result of the COVID-19 pandemic, being forced to carry out their activities online and therefore the Spanish education system had to exercise the teaching-learning process in a new scenario. In order to understand the perceptions of family members of Primary Education students, in private-concerted centers, this study disseminated a questionnaire during the month of March 2020. The results of the analysis of the data collected show that most families of the sample analyzed consider that the task load should vary according to the subjects, showing mostly patience in the resolution of doubts and developing good planning to continue the educational development of the course from home, manifestation that the time spent performing the tasks was extensive.

Keywords: primary education; homework; family; Covid-19; perceptions

\section{INTRODUCCIÓN}

En la actualidad, la situación pandémica del COVID-19 ha causado la necesidad de convivir con unas circunstancias excepcionales en el ámbito social y, en concreto, en el ámbito escolar (Yi et al., 2020), alterando las implicaciones y la vida de los ciudadanos en los diferentes escenarios en los que se ven inmersos para poder afrontar y dar respuesta a la crisis sanitaria (Hyland-Wood et al., 2021; 0'Sullivan et al., 2020). En este sentido, la enseñanza virtual ha adquirido gran protagonismo en la dependencia tecnológica, lo que ha dado lugar a cambios radicales en la organización y transferencia del conocimiento (Teräs et al., 2020).

Debido al proceso de globalización, accesibilidad, interactividad y flexibilidad de las Tecnologías de la Información y la Comunicación (TIC), la educación en tiempos de pandemia ha sufrido un incremento notorio en el uso de internet, ya que se ha podido constatar que la forma de trabajar, pensar y tomar decisiones está cambiando cada vez más (Garrote et al., 2018), viéndose afectada por el rol y las características modulares de las instituciones educativas (López et al., 2018). Sin embargo, a pesar de estas afirmaciones, en España existe una carencia de estudios que traten las dificultades en cuanto al desarrollo de las actividades por parte del alumnado al recibir la educación online durante el confinamiento (Díez-Gutiérrez y Gajardo-Espinosa, 2020; Muñoz y Lluch, 2020), y que éstos se enfoquen en la necesidad por educar en los tiempos que corren con el objetivo de renovar un mundo común (Beltran y Venegas, 2020; Covarrubias, 2021).

Por otro lado, la labor del docente en su faceta profesional debe incluir y adaptar el uso de las TIC en los contenidos curriculares propios de cada etapa educativa, pues el desarrollo de competencias hoy en día se enfoca en tender aspectos elementales sobre el alumnado los cuales den paso y favorezcan su propia formación (Escudero et al., 2018; Wang et al., 2020). A este respecto, las administraciones educativas han impulsado plataformas y espacios web que buscan dar soporte a los centros educativos, cuya finalidad era aunar recursos escolares que estuviera al uso de los docentes, los estudiantes y las familias, como ya lo estaban implantando en otros países (Wang et al., 2020; Zhang et al., 2020). No obstante, a pesar de estas iniciativas, la sociedad ha sido testigo de situaciones en donde la brecha digital fue expuesta, más aún, en que el alumnado se veía saturado en la realización de deberes (Palau et al., 2021), obligados a quedarse en casa y continuar las clases de forma online durante un largo periodo de confinamiento (Wang et al., 2020) y en entornos familiares que les ayudaban con los medios que podían (Brown et al., 2021). En esta línea y atendiendo a Cabrera (2020), esta situación en sí incrementa la desigualdad de oportunidades y, aunque sea un tema que afecta a todos, aquellos individuos que viven en lugares desfavorecidos pero que asisten a centros escolares públicos se encuentran con más problemas para hacer frente al nuevo entorno por sus propias condiciones personales y familiares.

La participación de los padres en el seguimiento de las tareas escolares en Educación Primaria se vuelve cada vez más importante, sobre todo en tiempos de COVID-19 (Feito, 2020). En consonancia, diferentes estudios han manifestado que la implicación e intervención entre padres y escuela provoca mejoras en variables mediadoras como es el caso del rendimiento académico y la motivación en el estudiantado (Distefano, et al., 2018; Ferraces et al., 2021). Así pues y de acuerdo con Núñez et al. (2012) el seguimiento de tareas escolares por parte 
de la familia refuerza la autorregulación y los hábitos de estudio en el alumnado. En la investigación de PérezDíaz et al. (2001) coinciden en que los estudiantes normalmente reciben ayuda de algún familiar: el 43\% de las familias consideran que casi siempre o siempre ayudan a sus hijos e hijas, y el 14\% los ayudan bastantes veces. Estos resultados disminuyen durante la Educación Secundaria a un 9\%, observando que la implicación familiar se reduce conforme aumenta la edad.

\section{OBJETIVOS DE LA INVESTIGACIÓN}

El presente estudio pretende conocer la situación educativa durante la cuarentena del COVID-19 analizando la percepción de las familias de estudiantes de Educación Primaria pertenecientes a centros privados-concertados durante el estado de alarma vivido en España en 2020.

\section{MUESTRA Y/O PARTICIPANTES}

Mediante un muestreo por conveniencia la investigación está constituida por un total de 93 familiares de estudiantes de Educación Primaria pertenecientes a diferentes centros privados-concertados de España. El 53\% de la muestra tiene edades comprendidas entre 36 y 45 años, un 30\% son mayores de 46 años y el 17\% restante tiene menos de 35 años.

\section{METODOLOGíA Y/O INSTRUMENTOS UTILIZADOS}

El diseño de la investigación es descriptivo, cuantitativo y transversal, empleando, para la recogida de datos un cuestionario, el cual fue previamente elaborado y sometido a juicio de expertos con la finalidad de que, académicos con amplia experiencia, evaluasen su validez de contenido.

La difusión del cuestionario se realizó mediante la plataforma Google Forms durante el mes de marzo de 2020, mientras que la sociedad se encontraba bajo el confinamiento ocasionado por el estado de alarma del COVID-19. Dicho cuestionario está compuesto por 25 ítems relacionados con aspectos contextuales, laborales y educativos para conocer la percepción de las familias de estudiantes de Educación Primaria.

\section{RESULTADOS ALCANZADOS}

Sobre los datos recogidos se han analizado 4 ítems del cuestionario. En el primer ítem, "Considero que todas las asignaturas deben tener la misma carga de tareas", la mayoría de la muestra ha respondido no estar conforme con la afirmación, encontrando que el 18.27\% está muy desacuerdo y el 52.68\% desacuerdo, frente a un $24.73 \%$ que está de acuerdo seguido de un bajo porcentaje, un 4.32\%, que manifiesta estar muy de acuerdo con lo afirmando en el ítem analizado tal y como se aprecia en la Figura 1.

Figura 1. Carga de tareas según asignaturas.

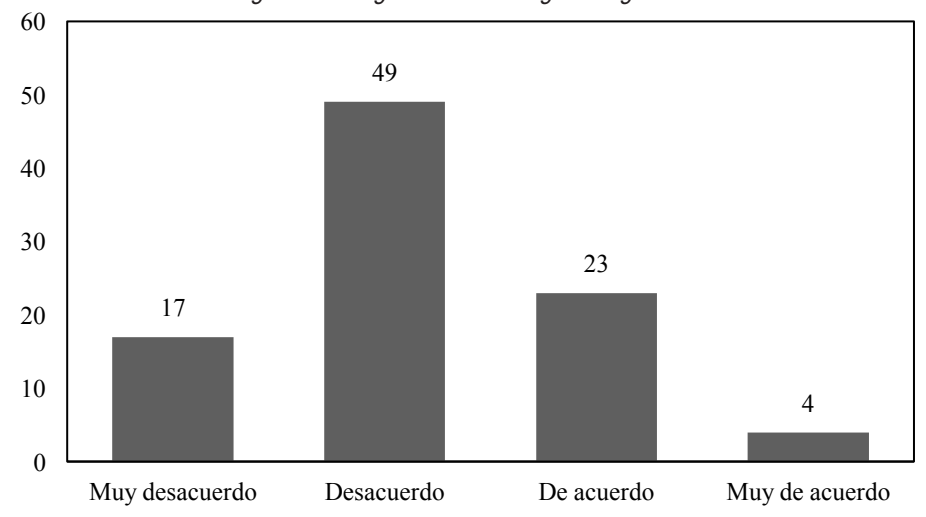


En relación a la paciencia manifestada mientras los familiares resuelven las dudas de los estudiantes de Educación Primaria durante el confinamiento, Ios datos recogidos en la Figura 2 reflejan que un alto porcentaje de la muestra está en consonancia con lo afirmado en el ítem "Cuando resuelvo dudas o doy explicaciones de ejercicios al estudiante muestro paciencia". La mayoría de los familiares se muestra de acuerdo con un $67.74 \%$, seguido de un $18.27 \%$ que manifiestan estar muy de acuerdo con la afirmación. Un bajo porcentaje, un $2.15 \%$ respondió estar muy desacuerdo junto con un $11.82 \%$ que, siguiendo la misma línea del porcentaje anterior, respondió estar desacuerdo.

Figura 2. Paciencia de los familiares en la resolución de dudas.

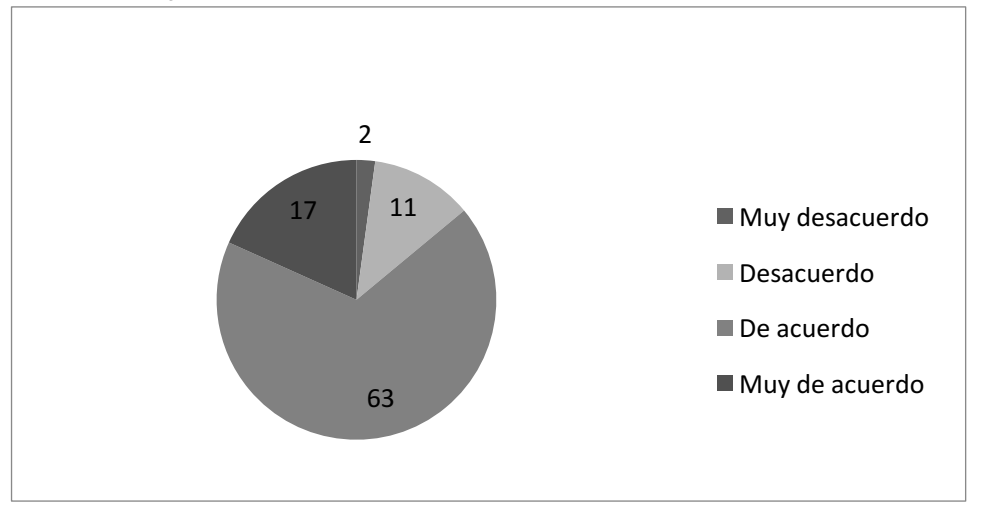

Sobre el tercer ítem planteado "Considero que el tiempo diario empleado en la realización de tareas es extenso", Ios familiares manifestaron en su mayoría que estaban de acuerdo con un $38.70 \%$ unido al $23.65 \%$ que respondieron estar muy de acuerdo, considerando que el tiempo empleado en las tareas escolares era extenso. Por otro lado, los familiares que se mostraron disonantes con la afirmación constituyen un 37.63\% sobre el total, aunando las respuestas en desacuerdo y muy desacuerdo como se aprecia en la Figura 3.

Figura 3. Consideración de emplear bastante tiempo en la realización de tareas.

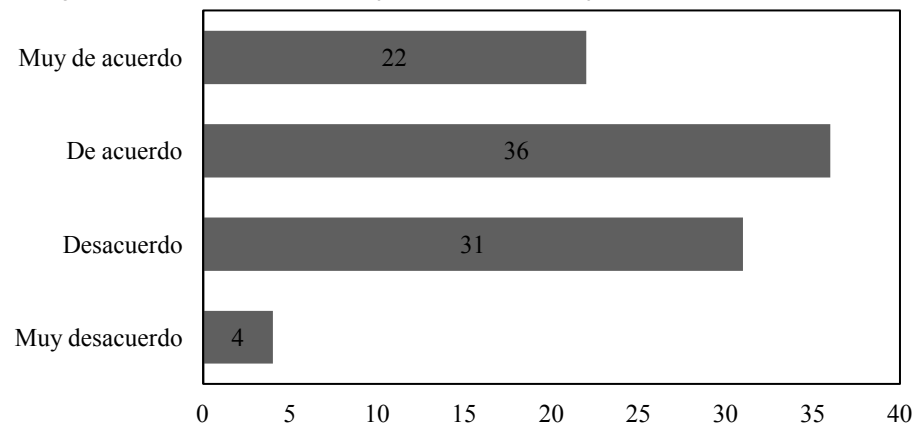

En lo que se refiere a la organización y planificación en el hogar para la realización de tareas y continuar con el curso de forma online, los datos obtenidos de la muestra encuestada muestran diferencias entre los familiares que se han organizado y los que no. En la Figura 4 se recogen las respuestas registradas, sobre las cuales el $58.06 \%$ está de acuerdo con la afirmación planteada, seguido del $32.25 \%$ que se manifiesta muy de acuerdo. A diferencia de los familiares que han planificado un horario escolar en el hogar encontramos que un bajo porcentaje no ha llevado a efecto lo expuesto con un $8.60 \%$ en desacuerdo y un $1.07 \%$ en muy desacuerdo. 
Figura 4. Buena organización en el hogar para la realización de tareas.

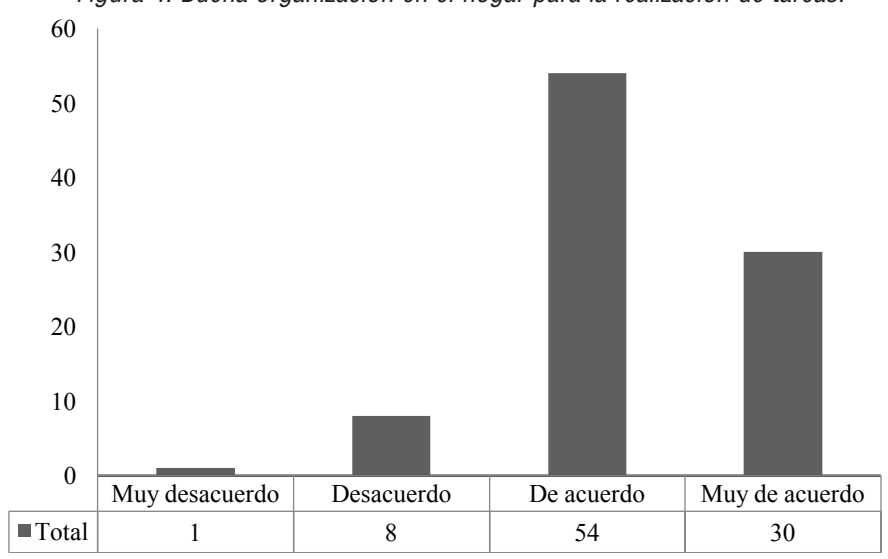

\section{DISCUSIÓN}

En los resultados se ha podido observar el análisis de 4 ítems del cuestionario final. En el primer ítem, la mayoría de los encuestados estaban de acuerdo con que no todas las asignaturas debían tener la misma carga de tareas. Esto puede ser debido a que cada materia precisa de unos tiempos y necesidades diferentes. En el segundo ítem, relacionado con la paciencia, un gran porcentaje de familiares aseguraban mostrar paciencia y tranquilidad cuando ayudaban y resolvían las dudas de los alumnos. Asimismo, Ferraces et al. (2021), afirman que el apoyo familiar influye en el rendimiento académico de los estudiantes. Por otro lado, el tercer ítem estuvo muy igualado en sus resultados, ya que los familiares de los alumnos de Educación Primaria tenían un porcentaje similar en estar o no de acuerdo con el tiempo que empleaban los alumnos cuando realizaban las tareas escolares. Quizás hay un desequilibrio en las horas y actividades que se le debe dedicar a cada asignatura. Además, de acuerdo con Feito (2020), hay un incremento desmesurado de los deberes en estudiantes de Educación Primaria y Secundaria. En el último ítem, sobre la importancia de la organización y planificación en el hogar mientras continuaba el curso de manera online, hemos podido observar que un alto porcentaje de familiares ha conseguido mantener un equilibrio entre las tareas del hogar y la ayuda a los alumnos sobre la formación online. Esto puede ser debido a que, en Educación Primaria Ios alumnos reciben más ayuda académica por parte sus padres y que conforme aumentan de edad y curso escolar, esta ayuda disminuye considerablemente (Pérez-Díaz et al., 2001).

\section{CONCLUSIONES}

El objetivo de este estudio era conocer la situación educativa durante la cuarentena del COVID-19. Para ello se analizó la percepción de las familias españolas de estudiantes de Educación Primaria pertenecientes a centros privados y concertados en marzo de 2020, durante el estado de alarma del COVID-19. En definitiva, el confinamiento y la pandemia han supuesto un cambio a grandes dimensiones en el ámbito escolar. La enseñanza presencial pasa a ser una enseñanza online y esto supone un incremento en la desigualdad de oportunidades educativas puesto que no todas las familias tienen a su alcance medios necesarios para que sus hijos se adhieran a la enseñanza virtual. Ya sea porque no dispongan de aparatos tecnológicos como ordenadores o tablets, 0 simplemente porque no tengan acceso a internet. Aun así, la pandemia y el estado de alarma han hecho que un alto porcentaje de padres y madres teletrabajen, lo que supone una implicación mayor en la enseñanza y aprendizaje de sus hijos. Sin embargo, se han visto implicados en una carga desmesurada de tareas que los niños debían realizar. Finalmente pensamos que durante el estado de alarma las familias han tenido un papel muy importante en la educación de los niños y han sabido cooperar, colaborar y ayudar a los centros educativos para que el alumnado pudiese terminar el cuso académico satisfactoriamente. 


\section{LIMITACIONES DEL ESTUDIO}

Este artículo proporciona información del cuestionario sobre la percepción que tienen las familias de los estudiantes de Educación Primaria durante el COVID-19. El estudio presenta las limitaciones típicas derivadas del sesgo de fiabilidad social, por lo que no podemos afirmar con exactitud que todas las respuestas sean reales. Otra de las limitaciones que cuenta el estudio es la pequeña muestra a la que se le pasa el cuestionario, ya que pensamos que debería ser mayor para tener unos resultados más concretos y exactos. Asimismo, se deberá tener cuidado si se decide utilizar el cuestionario en otros países, ya que, en términos de educación, cada país se rige por una normativa diferente.

\section{REFERENCIAS BIBLIOGRÁFICAS}

Beltrán, J., y Venegas, M. (2020). Educar en época de confinamiento: la tarea de renovar un mundo común. Revista de Sociología de la Educación, 13(2).

Brown, J., McLennan, C., Mercieca, D., Mercieca, D., Robertson, D., y Valentine, E. (2021). Technology as thirdspace: teachers in scottish schools engaging with and being challenged by digital technology in first COVID19 lockdown. Education Sciences, 11(3), 136. https://doi.org/10.3390/educsci11030136

Cabrera, L. (2020). Efectos del coronavirus en el sistema de enseñanza: aumenta la desigualdad de oportunidades educativas en España. Revista de Sociología de la Educación (RASE), 13(2), Especial COVID-19, 114-139. https://doi.org/10.7203/rase.13.2.17125

Covarrubias, L. Y. (2021). Educación a distancia: Transformación de los aprendizajes. Telos: Revista De Estudios Interdisciplinarios En Ciencias Sociales, 23(1), 150-160. https://doi.org/10.36390/telos231

Díez-Gutiérrez, E., y Gajardo-Espinoza, K. (2020). Educar y Evaluar en Tiempos de Coronavirus: la Situación en España. Multidisciplinary Journal of Educational Research, 10(2), 102-134. http://dx.doi.org/10.17583/remie.2020.5604

Distefano, R., Galisnsky, E., McClelland, M. M., Zelazo, P. D., y Carlson, S. M. (2018). Autonomy-supportive parenting and associations with child and parent executive function. Journal of Applied Developmental Psychology, 58, 77-85. https://doi.org/10.1016/j.appdev.2018.04.007

Escudero, J. M., Martínez-Domínguez, B., y Nieto, J. M. (2018). Las TIC en la formación continua del profesorado en el contexto español. Revista de Educación, 382, 57-80. https://doi.org/10.4438/1988-592X-RE-2018382-392

Feito, R. (2020). Este es el fin de la escuela tal y como la conocemos. Unas reflexiones en tiempo de confinamiento. Revista de Sociología de la Educación (RASE), 13(2), Especial COVID-19, 156-163. https://doi.org/10.7203/rase.13.2.17130

Ferraces, M. J., Lorenzo, M., Godás, A., y Santos, M. A. (2020). Students' mediator variables in the relationship between family involvement and academic performance: effects of the styles of involvement. Psicología Educativa, 27(1), 85-92. https://doi.org/10.5093/psed2020a19

Garrote, D., Jiménez-Fernández, S., y Serna, R. (2018). Gestión del tiempo y uso de las TIC en estudiantes universitarios. Píxel-Bit, 53, 109-121. https://doi.org/10.12795/pixelbit.2018.i53.07

Hyland-Wood, B., Gardner, J., Leask, L., y Ecker, U. (2021). Toward effective government communication strategies in the era of COVID-19. Humanities and Social Sciences Commnications, 8(30). https://doi.org/10.1057/s41599-020-00701-w

López, L., López, B., y Prieto, E. (2018). Tendencias innovadoras en la formación on-line. La oferta web de postgrados e-learning y blended learning en España. Píxel-Bit, 53, 1-15. https://doi.org/10.12795/pixelbit.2018.i53.06

Muñoz Moreno, J. L., y Lluch Molins, L. (2020). Educación y Covid-19: Colaboración de las Familias y Tareas Escolares. Revista Internacional De Educación Para La Justicia Social, 9(3), 1-17.

Núñez, J. C., Suárez, N., Fernández, M., Cerezo, R., Rodríguez, C., y Rosario, P. (2012). Tareas para casa, impli- 
cación familiar y rendimiento académico. Aula Abierta, 40(1), 73-84.

O'Sullivan, D., Rahamathulla, M., y Pawar, M. (2020). The impact and implications of COVID-19: an Australian perspective. The International Journal of Community and Social Development, 2(2), 134-151. https://doi.org/10.1177/2516602620937922

Palau, R., Fuentes, M., Mogas, J., y Cebrián, G. (2020). Analysis of the implementation of teaching and learning processes at Catalan schools during the Covid-19 lockdown. Technology, Pedagogy and Education, 1-17. https://doi.org/10.1080/1475939X.2020.1863855

Pérez-Díaz, V., Rodríguez, J., y Sánchez, L. (2001). La familia española ante la educación de sus hijos. Barcelona: Fundación La Caixa.

Teräs, M., Suoranta, H., Teräs, H., y Curcher, M. (2020). Post-Covid-19 education and education technology "solutionism": a seller s market. Postdigital Science and Education, 2, 863-878. https://doi.org/10.1007/s42438-020-00164-X

Wang, C., Cheng, Z., Yue, X. G., y McAleer, M. (2020). Risk Management of COVID-19 by Universities in China. Journal of Risk and Financial Management, 13(2), 36. http://dx.doi.org/10.3390/jrfm13020036

Wang, G., Zhang, Y., Zhao, J., Zhang, J., y Jiang, F. (2020). Mitigate the effects of home confinement on children during the COVID-19 outbreak. The Lancet, 395(10228), 945-947. https://doi.org/10.1016/S01406736(20)30547-X

Yi, Y., Lagniton, P., Ye, S., Li, E., y Xu, R. H. (2020). covid-19: what has been learned and to be learned about the novel coronavirus disease. International Journal of Biological Sciences, 16, 10, 1753-1766.

Zhang, Y., y Ma, Z. F. (2020). Impact of the COVID-19 Pandemic on Mental Health and Quality of Life among Local Residents in Liaoning Province, China: A Cross-Sectional Study. International Journal of Environmental Research and Public Health, 17(7), 2381. https://doi.org/10.3390/ijerph17072381 
